\title{
Ocular Manifestations of Hospitalized COVID-19 Patients in a Tertiary Care Academic Medical Center in the United States: A Cross-Sectional Study
}

This article was published in the following Dove Press journal:

Clinical Ophthalmology

\author{
Yilin Feng' \\ Jemin Park' \\ Yunshu Zhou (D) \\ Stephen T Armenti ${ }^{1}$ \\ David C Musch ${ }^{1,2}$ \\ Shahzad I Mian' \\ 'Department of Ophthalmology and \\ Visual Sciences, University of Michigan, \\ Kellogg Eye Center, Ann Arbor, MI, USA; \\ ${ }^{2}$ Department of Epidemiology, University \\ of Michigan, Ann Arbor, MI, USA
}

Purpose: Studies have identified a wide range of ocular signs and symptoms in coronavirus disease 2019 (COVID-19) patients; however, these studies were often conducted outside of the United States. We aim to investigate the ocular manifestations of hospitalized COVID-19 patients at a tertiary care medical center in the United States.

Patients and Methods: A retrospective, cross-sectional study was conducted on individuals aged 18 and over who were hospitalized for COVID-19 between March 10, 2020 and April 13, 2020. The electronic health record was reviewed for all patients, and a follow-up phone survey was conducted on patients who were discharged home. Data on patient history, physical exam, laboratory results, and hospital disposition were collected and analyzed.

Results: A total of 400 patients were included. The mean patient age was 61.7 years (SD $15.5)$ and $233(58.3 \%)$ were males. Ocular signs and symptoms were noted in $38(9.5 \%)$ patients. The most common ocular abnormality was conjunctival injection, followed by vision changes and ocular irritation. Among the 38 patients, $30(79.0 \%)$ developed ocular involvement prior to day 30 of onset of their COVID symptoms. Univariate analysis showed that age, gender, ocular history, fever, mechanical ventilation, and increasing inflammatory markers were not significantly associated with the presence or development of ocular symptoms.

Conclusion: In this study, 9.5\% of hospitalized COVID-19 patients exhibited ocular signs and symptoms. Factors associated with severe systemic COVID-19 disease were not associated with developing ocular abnormalities. The rate of ocular manifestations of COVID-19 should not be ignored, and thus physicians should routinely evaluate for ocular involvement in hospitalized COVID-19 patients.

Keywords: COVID-19, conjunctivitis, ocular manifestations, ocular surface

\section{Introduction}

Severe acute respiratory syndrome coronavirus 2 (SARS-CoV-2), the pathogen causing coronavirus disease 2019 (COVID-19), is primarily transmitted through the contact of mucous membranes with infectious respiratory droplets. Recent evidence suggests that ocular transmission is possible, as SARS-CoV-2 was shown to replicate in conjunctival tissue and conjunctival secretions have tested positive for SARS-CoV-2 RNA using reverse transcriptase-polymerase chain reaction (RT-PCR). ${ }^{1-4}$ Interestingly, there appears to be no direct correlation between
Professor of Ophthalmology and Visual Sciences, University of Michigan, Kellogg Eye Center, 1000 Wall St, Ann Arbor, MI, 48105, USA

Tel + I 734-763-5904

Email smian@med.umich.edu 
ocular symptoms and the presence of virus in conjunctival secretions, as patients with ocular manifestations can test negative for conjunctival RT-PCR, and vice versa. ${ }^{2,3,5}$

Recent reports have suggested that ocular symptoms may be an initial manifestation of COVID-19 infection, highlighting the importance of understanding how COVID-19 can affect the eyes. ${ }^{6-8}$ The reported prevalence of ocular signs and symptoms in COVID-19 patients is variable, with a recent meta-analysis of three studies showing that the overall rate of conjunctivitis is $1.1 \%$ in COVID-19 patients, with $3 \%$ and $0.7 \%$ in severe and non-severe COVID-19 patients, respectively. ${ }^{9}$ There is additional evidence indicating that patients with ocular involvement may have more severe disease. ${ }^{4}$ Currently, there are no studies that assess the ocular manifestations of hospitalized COVID-19 patients at a tertiary care medical center in the United States. It is important to fully understand the signs and symptoms of the disease and its modes of transmission in order to identify new cases to decrease virus transmission. Thus, in this study, we aim to investigate the ocular manifestations of hospitalized COVID-19 patients at a single tertiary care academic medical center in the United States.

\section{Patients and Methods}

This study was approved by the Institutional Review Board at the University of Michigan. Patient consent was obtained for all participants in the phone survey, and patient consent was waived by the Institutional Review Board for electronic medical record review given its classification as secondary research for which consent is not required. All patient-protected health information remained confidential in this study. DataDirect, a self-serve tool enabling access to clinical data was used to search for all patients aged 18 and over who were admitted to Michigan Medicine for COVID-19 between March 10, 2020 and April 13, 2020. Patients were only included if they had a positive nasopharyngeal PCR for SARS-CoV-2 and if COVID-19 was the primary indication for admission. For each patient, two reviewers extracted the following information from the electronic health record: age, gender, date of birth, date of service, length of stay, past ocular history, ocular medications, day of symptom onset, ocular symptoms, ocular physical exam, treatments for ocular symptoms, ventilation status, and disposition. The ocular exam was not performed by an ophthalmologist unless an ophthalmic evaluation was requested by the primary team, which was rare. In addition, temperature, c-reactive protein (CRP), D-dimer, ferritin, and absolute lymphocyte count (ALC) were recorded when available. All patients who were discharged home and were able to self-consent were followed up with a phone survey by a designated study team member. In the phone survey, patients were asked if they experienced any eye symptoms such as red eyes, eye discharge, eye swelling, or vision changes during the course of their COVID-19 illness, the day of onset of any ocular symptoms, and any treatments received.

Descriptive statistics were generated for the entire study cohort and by status of having ocular signs and symptoms during the course of COVID-19. Differences between groups were assessed using the chi-square test or Fisher exact test for categorical variables, and the Wilcoxon rank-sum test for continuous variables. Univariate logistic regression models were employed to assess the associations between the outcome of developing ocular symptoms and the factors of interest. Odds ratios, 95\% confidence intervals, and p-values were reported. A $p$-value $<0.05$ was used to indicate statistical significance. All analyses were conducted using SAS 9.4 (SAS Institute, Cary, NC, USA).

\section{Results}

A total of 400 patients were included in the study. The mean age was 61.7 years old (SD 15.5) and 233 (58.3\%) were male (Table 1). The average hospital length of stay was 15.1 days (SD 14.9). Seventy-seven (19.3\%) patients had prior ocular history documented in their medical record, 259 (64.8\%) patients experienced a fever during their admission, and 157 (39.3\%) patients required mechanical ventilation. Two hundred and fifty-seven $(64.3 \%)$ patients were discharged home, 71 (17.8\%) were discharged to a rehabilitation facility, and 72 (18.0\%) were transitioned into hospice care or passed away. Following discharge, 234 (58.5\%) patients were called, and 113 $(28.3 \%)$ patients consented to participate in the phone survey.

Based on electronic health record review alone, 19 patients exhibited ocular signs and symptoms. An additional 19 patients from the phone survey reported ocular abnormalities that were not documented in the electronic health record, including floaters and vision changes, among others. In total, $38(9.5 \%)$ patients exhibited ocular signs and symptoms in this study. Among the 38 patients, $30(79.0 \%)$ developed ocular abnormalities prior to day 30 of onset of COVID symptoms. Seven patients (18.4\%) 
Table I Characteristics of Hospitalized Patients with COVID-19

\begin{tabular}{|c|c|c|c|c|}
\hline & Overall $(\mathrm{N}=400)$ & With Ocular Symptoms $n=38$ & No Ocular Symptoms $n=362$ & P-value \\
\hline Age, mean (SD), y & $61.7(15.5)$ & $59.2(16.0)$ & $61.9(15.4)$ & 0.2529 \\
\hline Male, No. (\%) & $233(58.3)$ & $26(68.4)$ & $207(57.2)$ & 0.1814 \\
\hline Fever, No. (\%) & $259(64.8)$ & $29(76.3)$ & $230(63.5)$ & 0.1167 \\
\hline Prior ocular history, No. (\%) & $77(19.3)$ & II (29.0) & $66(18.2)$ & 0.1110 \\
\hline Mechanical ventilation, No. (\%) & $157(39.3)$ & $18(47.4)$ & $139(38.4)$ & 0.2813 \\
\hline \multicolumn{5}{|l|}{ Disposition, No. (\%) } \\
\hline Home & $257(64.3)$ & 27 (7I.I) & $230(63.5)$ & 0.6249 \\
\hline Facility & $71(17.8)$ & $6(15.8)$ & $65(18.0)$ & \\
\hline Hospice/Deceased & $72(18.0)$ & $5(13.2)$ & $67(18.5)$ & \\
\hline $\mathrm{CRP}^{\mathrm{a}}, \mathrm{mg} / \mathrm{dL}$ & $9.5(5.7,14.5)$ & $9.6(6.7,14.5)$ & $9.4(5.6,14.6)$ & 0.5265 \\
\hline D-Dimer ${ }^{\mathrm{a}}, \mathrm{mg} / \mathrm{L}$ & I.8 $(0.9,5.3)$ & I.8 (I.0, 4.6) & I.8 $(0.9,5.3)$ & 0.9919 \\
\hline Ferritin $^{\mathrm{a}}, \mathrm{ng} / \mathrm{mL}$ & $942.1(435.9,1499.4)$ & $777.4(532.1,1646.2)$ & $944.7(435.9,1473.8)$ & 0.9008 \\
\hline $\mathrm{ALC}^{\mathrm{a}}, \mathrm{K} / \mathrm{uL}$ & I.I $(0.8$, I.5) & $1.0(0.8,1.5)$ & I.I $(0.9,1.5)$ & 0.5400 \\
\hline
\end{tabular}

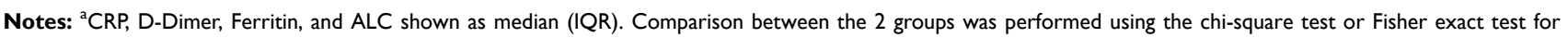
categorical variables and the Wilcoxon rank-sum test for continuous variables.

Abbreviations: COVID-19, coronavirus disease 2019; SD, standard deviation; CRP, C-reactive protein; ALC, absolute lymphocyte count.

reported ocular symptoms as one of the initial manifestations of COVID-19, and on average, patients developed ocular signs and symptoms on day 17 (SD 15) of onset of COVID-19 symptoms. There was no difference in the age, gender, presence of prior ocular history, fever during hospitalization, mechanical ventilation, and inflammatory marker levels between patients with and without ocular signs and symptoms. The most common ocular abnormality was conjunctival injection, followed by vision changes and ocular irritation (Table 2). Univariate analysis revealed that age, gender, presence of prior ocular history, fever during hospitalization, mechanical ventilation, and increasing inflammatory markers were not significantly associated with the presence or development of ocular symptoms (Table 3). A multivariable logistic regression was not conducted as no covariate demonstrated a statistically significant association with univariate analysis.

\section{Discussion}

To our knowledge, this is the first study that investigates the ocular manifestations of hospitalized COVID-19 patients at a tertiary care academic medical center in the United States. Prior published reports on the ocular manifestations of COVID-19 patients have mostly originated from Asia and Europe. Our study demonstrates that $9.5 \%$ of hospitalized patients exhibited ocular abnormalities. This rate is similar to a recent report of 127 COVID-19 cases from India, which found that $9.45 \%$ of patients had ocular complaints, although patients from this study only had mild cases of COVID19. ${ }^{10}$ Similar to previous studies, common ocular manifestations in our cohort were signs and symptoms consistent with conjunctivitis, such as conjunctival injection

Table 2 Distribution of Ocular Signs and Symptoms

\begin{tabular}{|c|c|}
\hline Signs and Symptoms ${ }^{a}$ & With Ocular Symptoms (\%) \\
\hline Overall & $38(9.5)$ \\
\hline Conjunctival injection & $15(39.5)$ \\
\hline Vision changes & $9(23.7)$ \\
\hline Irritation & $8(2 I . I)$ \\
\hline Discharge & $7(18.4)$ \\
\hline Itching & $3(7.9)$ \\
\hline Scleral hemorrhage & $2(5.3)$ \\
\hline Other $^{\mathrm{b}}$ & $6(15.8)$ \\
\hline
\end{tabular}

Notes: ${ }^{a}$ Some patients experienced more than one ocular sign and symptom. bOther includes floaters, scleral edema, eye bubble, eyelid swelling, periorbital edema, exposure keratopathy with corneal ulcer. 
Table 3 Univariate Analysis of Factors Associated with the Presence of Ocular Symptoms

\begin{tabular}{|c|c|}
\hline & $\begin{array}{l}\text { Odds Ratio ( } 95 \% \\
\text { Confidence Interval) }\end{array}$ \\
\hline $\mathrm{Age}^{\mathrm{a}}$ & $0.95(0.85,1.05)$ \\
\hline Female & $0.62(0.30,1.26)$ \\
\hline Fever & $1.85(0.85,4.03)$ \\
\hline Prior ocular history & I.83 $(0.86,3.87)$ \\
\hline Mechanical ventilation & I.44 $(0.74,2.83)$ \\
\hline \multicolumn{2}{|l|}{ Disposition } \\
\hline Facility vs home & $0.79(0.31,1.99)$ \\
\hline Hospice/Deceased vs home & $0.64(0.24,1.72)$ \\
\hline $\mathrm{CRP}, \mathrm{mg} / \mathrm{dL}^{\mathrm{a}}$ & I.0I $(0.96,1.05)$ \\
\hline D-Dimer, $\mathrm{mg} / \mathrm{L}^{\mathrm{a}}$ & $1.00(0.95,1.05)$ \\
\hline Ferritin, $\mathrm{ng} / \mathrm{mL}^{\mathrm{a}}$ & $1.00(0.93,1.07)$ \\
\hline $\mathrm{ALC}, \mathrm{K} / \mathrm{uL}^{\mathrm{a}}$ & I.0I $(0.79,1.3 \mathrm{I})$ \\
\hline
\end{tabular}

Notes: ${ }^{a}$ Odds ratio estimate was calculated based on increase of 5 -year increments for age, I unit increments for CRP and D-Dimer, 250 unit increments for ferritin, and 0.5 unit increments for ALC.

Abbreviations: CRP, C-reactive protein; ALC, absolute lymphocyte count.

and discharge. However, vision changes were also noted to be a prominent symptom, found in $23.7 \%$ of patients with ocular abnormalities. While the etiology of vision changes was not elucidated due to limited electronic record documentation and details from patient report, possible mechanisms could include ocular surface abnormalities or retinal pathology, as previously reported. ${ }^{11}$

In this study, we assessed whether there were significant differences between patients with and without ocular abnormalities. Previous studies have suggested that patients with ocular symptoms have higher CRP and lymphocyte counts and were also more likely to be older and have fever, suggesting that patients with ocular symptoms were likely to have more severe COVID-19 disease. ${ }^{3,12}$ However, our cohort did not show this association. To further address this question, we also used mechanical ventilation and other inflammatory markers such as D-dimer and ferritin as proxies for disease severity and found no difference in these factors between patients with and without ocular symptoms. The difference in the results between our study and prior studies could be due to our larger patient cohort, differences in COVID-19 severity, and/or variations in data collection and patient setting, as prior reports were mostly conducted with cohorts outside of the United States.

In addition to comparing patients with and without ocular symptoms, we also sought to determine whether certain factors may be associated with the development of ocular signs and symptoms in COVID-19. Our univariate analysis demonstrated that demographic characteristics such as age and gender did not increase the odds of developing ocular abnormalities among COVID-19 patients. In addition, there was no association between the presence of ocular history and development of ocular manifestations. We recognize that a variety of ocular events can trigger the "prior ocular history" variable and thus this is a variable that lacks specificity, which is a limiting factor in its interpretation. Furthermore, markers of disease severity such as fever, mechanical ventilation, and increasing inflammatory markers were also not associated with the development of ocular symptoms.

Our study provides novel insight into the onset of ocular signs and symptoms in COVID-19 patients. Of patients in our study, $18.4 \%$ reported that ocular signs and symptoms were one of the initial manifestations of their disease. On average, however, patients developed ocular symptoms on day 17 of COVID-19 symptoms. This is consistent with previous reports that suggest that ocular symptoms can be the initial manifestation of disease. ${ }^{13-15}$ It is not surprising that there are ocular manifestations of COVID-19, as research has identified the presence of ACE2 and TMPRSS2 receptors, which the SARS-CoV-2 virus uses to gain entry in tissues, in the eye. ${ }^{16,17}$ In addition, there is growing evidence that SARSCoV-2 RNA can be isolated from ocular tissue, including the conjunctiva, cornea, and vitreous. ${ }^{18}$ However, as our study suggests, only a minority of patients with COVID19 exhibit ocular symptoms. This is likely due to the presence of innate ocular protective mechanisms against viral infections. The eye contains natural physiological and anatomical factors, such as the presence of eyelashes, which protect the eye from microdroplets carrying viral particles. ${ }^{19}$ Nonetheless, these protective mechanisms are not completely foolproof, and given the possible ocular transmission of SARS-CoV-2, methods have been proposed for ophthalmology practices to reduce possible transmission. ${ }^{20-22}$ Thus, physicians caring for COVID-19 patients should remain vigilant regarding monitoring for ocular signs and symptoms, as patients recovering from 
COVID-19 may also develop ocular symptoms that may be related to SARS-CoV-2.

In this study, 50\% (19) of the patients who developed ocular signs and symptoms were captured through phone call follow-up only, as these ocular abnormalities were not documented in the electronic health record. It is possible that physicians caring for hospitalized COVID-19 patients may have missed these concerns during their evaluation. When caring for COVID-19 patients, physicians may often elect to conduct a focused history over the phone or examine the patient from outside the room due to the risk of transmission and the related need to conserve personal protective equipment. However, emerging evidence suggests that extrapulmonary manifestations of COVID-19 are frequently encountered and should not be ignored. ${ }^{23}$ While it is possible that the ocular history or exam was conducted but not documented, complete documentation of the electronic health record should be emphasized for the provision of comprehensive patient care.

Our study has several limitations. First, the majority of patients in the study were not examined by an ophthalmologist and documented ocular exams were most often performed by emergency medicine, internal medicine, or anesthesiology physicians. The patients included in the study were admitted primarily for COVID-19, and the secondary ocular symptoms as a result were rarely significant enough to warrant ophthalmology consultation. Due to concern for viral transmission and efforts to protect healthcare workers, we reviewed the electronic health records and contacted patients by phone to determine if ocular manifestations were present as opposed to performing ocular exams when clinically not indicated. Due to this limitation, it is likely that minor ophthalmic events were missed or not reported given lack of detailed ophthalmic exams. In order to maintain the integrity of the data, the original descriptions of ocular abnormalities as documented and reported were unchanged. For example, scleral hemorrhage was documented by non-ophthalmology providers in two patients (Table 2), which likely represented subconjunctival hemorrhage. Similarly, scleral edema was documented by a provider, which likely represented chemosis. As a retrospective study, a causal association cannot be made between the presence of ocular symptoms and COVID-19. One patient who was examined by an ophthalmologist was diagnosed with exposure keratopathy with corneal ulcers, which likely was a result of critical illness and prolonged intensive unit care stay, and not SARS-CoV
-2. However, our observations are clinically important and suggest that in addition to direct ocular manifestations caused by SARS-CoV-2, indirect ocular symptoms can also occur in COVID-19 patients, warranting thorough eye exams by providers. Our study was also subject to selection bias, as we were only able to conduct follow-up phone calls to patients who were discharged home. These patients were likely to have less severe illness. It is possible that the rate of ocular manifestations may increase had we been able to follow-up with more patients, as our study suggests that electronic record review alone may be insufficient to capture all of the ocular signs and symptoms experienced by patients. The patients included in the study were hospitalized at a large academic tertiary care and referral center, and thus our patient population differs from those at a community or local hospital, potentially limiting generalizability. Finally, the small number of COVID-19 patients who demonstrated ocular signs and symptoms $(n=38)$ likely limited our power to detect associations with factors of clinical relevance.

\section{Conclusion}

As the pandemic continues to affect the United States, it is imperative for physicians and the general public alike to understand the manifestations of COVID-19. Given previous detection of viral RNA in conjunctival secretions, transmission of SARS-CoV-2 through ocular secretions cannot be ignored. Overall, our results suggest that the rate of ocular signs and symptoms of COVID-19 should not be ignored, although factors associated with the development of ocular signs and symptoms are still unclear. Additional predictors of developing ocular symptoms from COVID-19 remain to be identified in order to accurately inform the public on the manifestations of the disease, as well as understand the need for ocular personal protective equipment for our frontline healthcare workers.

\section{Acknowledgments}

We'd like to thank Dr. Lindsay Petty from the University of Michigan Department of Infectious Diseases for her input on the study design.

\section{Funding}

This work was funded by the University of Michigan Medical School Capstone for Impact Grant.

\section{Disclosure}

The authors report no conflicts of interest in this work. 


\section{References}

1. Hui KPY, Cheung MC, Perera RAPM, et al. Tropism, replication competence, and innate immune responses of the coronavirus SARS-CoV-2 in human respiratory tract and conjunctiva: an analysis in ex-vivo and in-vitro cultures. Lancet Respir Med. 2020;8 (7):687-695. doi:10.1016/S2213-2600(20)30193-4

2. Liang $\mathrm{L}, \mathrm{Wu}$ P. There may be virus in conjunctival secretion of patients with COVID-19. Acta Ophthalmol. 2020;98(3):223. doi:10.1111/aos.14413

3. Wu P, Duan F, Luo C, et al. Characteristics of ocular findings of patients with coronavirus disease 2019 (COVID-19) in Hubei Province, China. JAMA Ophthalmol. 2020;138(5):575-578. doi:10.1001/jamaophthalmol.2020.1291

4. Arora R, Goel R, Kumar S, et al. Evaluation of SARS-CoV-2 in tears of patients with moderate to severe COVID-19. Ophthalmology. 2020. doi:10.1016/j.ophtha.2020.08.029

5. Yu Jun IS, Anderson DE, Zheng Kang AE, et al. Assessing viral shedding and infectivity of tears in coronavirus disease 2019 (COVID-19) Patients. Ophthalmology. 2020;127(7):977-979. doi:10.1016/j.ophtha.2020.03.026

6. Cheema M, Aghazadeh H, Nazarali S, et al. Keratoconjunctivitis as the initial medical presentation of the novel coronavirus disease 2019 (COVID-19). Can J Ophthalmol. 2020;55(4):e125-e129. doi:10.1016/j.jcjo.2020.03.003

7. Lu C, Liu X, Jia Z. 2019-nCoV transmission through the ocular surface must not be ignored. Lancet. 2020;395(10224):e39. doi:10.1016/S0140-6736(20)30313-5

8. Nuzzi F, Carucci LL, Tripoli F. COVID-19 and ocular implications: an update. $J$ Ophthalmic Inflamm Infect. 2019;9(1):17.

9. Loffredo L, Pacella F, Pacella E, et al. Conjunctivitis and COVID-19: a meta-analysis. J Med Virol. 2020;92(9):1413-1414. doi:10.1002/ jmv. 25938

10. Sindhuja K, Lomi N, Asif M, Tandon R. Clinical profile and prevalence of conjunctivitis in mild COVID-19 patients in a tertiary care COVID-19 hospital: a retrospective cross-sectional study. Indian J Ophthalmol. 2020;68(8):1546. doi:10.4103/ijo.IJO_1319_20

11. Marinho PM, Marcos AAA, Romano AC, Nascimento H, Belfort R. Retinal findings in patients with COVID-19. Lancet. 2020;395 (10237):1610. doi:10.1016/S0140-6736(20)31014-X
12. Bostanci Ceran B, Ozates S. Ocular manifestations of coronavirus disease 2019. Graefes Arch Clin Exp Ophthalmol. 2020;258 (9):1959-1963. doi:10.1007/s00417-020-04777-7

13. Daruich A, Martin D, Bremond-Gignac D. Ocular manifestation as first sign of coronavirus disease 2019 (COVID-19): interest of telemedicine during the pandemic context. J Fr Ophtalmol. 2020;43 (5):389-391. doi:10.1016/j.jfo.2020.04.002

14. Khavandi S, Tabibzadeh E, Naderan M, Shoar S. Corona virus disease-19 (COVID-19) presenting as conjunctivitis: atypically high-risk during a pandemic. Cont Lens Anterior Eye. 2020;43 (3):211-212. doi:10.1016/j.clae.2020.04.010

15. Chen L, Deng C, Chen X, et al. Ocular manifestations and clinical characteristics of 535 cases of COVID-19 in Wuhan, China: a cross-sectional study. Acta Ophthalmol (Copenh). 2020;98(8). doi:10.1111/aos.14472.

16. Zhou L, Xu Z, Castiglione GM, Soiberman US, Eberhart CG, Duh EJ. ACE2 and TMPRSS2 are expressed on the human ocular surface, suggesting susceptibility to SARS-CoV-2 infection. Ocul Surf. 2020;18(4):537-544. doi:10.1016/j.jtos.2020.06.007

17. Sitaula RK, Khatri A, Janani MK, et al. Unfolding COVID-19: lessons-in-learning in Ophthalmology. Clin Ophthalmol. 2020;14:2807-2820. doi:10.2147/OPTH.S259857

18. Sawant OB, Singh S, Wright III RE, et al. Prevalence of SARS-CoV2 in human post-mortem ocular tissues. Ocul Surf. 2021;19:322-329. doi:10.1016/j.jtos.2020.11.002

19. Zimmerman K, Kearns F, Tzekov R. Natural protection of ocular surface from viral infection - a hypothesis. Med Hypotheses. 2020;143:110082. doi:10.1016/j.mehy.2020.110082

20. Sadhu S, Agrawal R, Pyare R. COVID-19: limiting the risks for eye care professionals. Ocul Immunol Inflamm. 2020;28(5):714-720. doi:10.1080/09273948.2020.1755442

21. Limbu B, Khatri A, Joshi P. COVID-19 preferred practice pattern for eye care professional - an initiative by Nepal Ophthalmic Society. Nepal J Ophthalmol. 2020;12(2):176-190.

22. Feng Y, Armenti ST, Mian SI. COVID-19 and the eye: a comprehensive review of the literature. Int Ophthalmol Clin. 2021;61(1):1-14. doi:10.1097/IIO.0000000000000339

23. Gupta A, Madhavan MV, Sehgal K, et al. Extrapulmonary manifestations of COVID-19. Nat Med. 2020;26(7):1017-1032.
Clinical Ophthalmology

\section{Publish your work in this journal}

Clinical Ophthalmology is an international, peer-reviewed journal covering all subspecialties within ophthalmology. Key topics include: Optometry; Visual science; Pharmacology and drug therapy in eye diseases; Basic Sciences; Primary and Secondary eye care; Patient Safety and Quality of Care Improvements. This journal is indexed on PubMed
Central and CAS, and is the official journal of The Society of Clinical Ophthalmology (SCO). The manuscript management system is completely online and includes a very quick and fair peer-review system, which is all easy to use. Visit http://www.dovepress.com/ testimonials.php to read real quotes from published authors. 